\title{
Surveying the Delivery Methods of CRISPR/Cas9 for ex vivo Mammalian Cell Engineering
}

\author{
William J. Kelton ${ }^{\mathrm{ab}}$, Theresa Pesch ${ }^{\mathrm{ab}}$, Stefan Matile ${ }^{\mathrm{bc}}$, and Sai T. Reddy ${ }^{\star a b}$
}

\begin{abstract}
The simplicity of the CRISPR/Cas9 technology has been transformative in making targeted genome editing accessible for laboratories around the world. However, due to the sheer volume of literature generated in the past five years, determining the best format and delivery method of CRISPR/Cas9 components can be challenging. Here, we provide a brief overview of the progress that has been made in the ex vivo genome editing of mammalian cells and summarize the key advances made for improving efficiency and delivery of CRISPR/ Cas9 in DNA, RNA, and protein form. In particular, we highlight the delivery of Cas9 components to human cells for advanced genome editing applications such as large gene insertion.
\end{abstract}

Keywords: Cas9 $\cdot$ CRISPR $\cdot$ Genome editing

\section{Introduction}

The recent discovery of nucleases with programmable specificity has led to a revolution in targeted genome engineering. Foremost among these is the CRISPR/ Cas9 system, which uses an RNA guide sequence (gRNA) to direct the Cas9 nuclease to complementary regions throughout the genome, leading to the generation of double-stranded breaks (DSBs). ${ }^{[1]}$ The rapid adoption of CRISPR/Cas9 is principally due to the simplicity of the system, especially since specialized protein engineering expertise is not required, in contrast to zinc-finger nuclease (ZNFs) and transcription activator-like effector nuclease (TALENs) technologies. In nature, CRISPR/Cas9 functions as a form of adaptive microbial immunity against bacteriophage infection. ${ }^{[2,3]}$ CRISPR RNA (crRNA) and trans-activating crRNA (tracrRNA) are generated separately but have been cleverly repurposed into a single chimeric gRNA $^{[4]}$ (Fig. 1). Thus, Cas9 can be retargeted simply by the synthesis of new complementary gRNAs. However, for gRNA binding to trigger Cas9 cleavage, there is also the need for a protospacer-ad-

${ }^{*}$ Correspondence: Prof. Dr. S. T. Reddyab

E-Mail: sai.reddy@ethz.ch

aDepartment of Biosystems Science and Engineering ETH Zürich, Basel

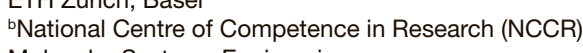

Molecular Systems Engineering

'Department of Organic Chemistry

University of Geneva, Geneva jacent motif (PAM), which is recognized by residues in the Cas9 protein (e.g. NGG for $S$. pyogenes Cas9) and thus required for generation of DSBs by the RuvC and $\mathrm{HNH}$ nuclease domains. ${ }^{[5]}$ The formation of double-stranded genomic breaks strongly activates DNA damage repair pathways such as non-homologous end joining (NHEJ), microhomology-mediated end joining (MMEJ), and homology-directed repair (HDR), all of which can be exploited for genome editing applications. In the case of NHEJ, error-prone base insertions and deletions (Indels) result in frequent frameshift mutations and premature stop codons, that are typically used for gene knockout. ${ }^{[6]}$ However, it has been shown that insertion of an exogenous DNA fragment can also take place at a targeted DSB via NHEJ.[7] MMEJ facilitates insertion in a similarly imprecise manner but uses short regions of microhomology ( $\geq 2 b p$ ), supplied by an exogenously derived donor template, to align broken DNA ends at the site of a DSB. [8] In contrast to these error-prone repair pathways, DSB repair via HDR provides precise sequence substitution in the presence of a homologous repair template, ${ }^{[9]}$ albeit at a much lower frequency than NHEJ or MMEJ in mammalian cells. ${ }^{[10]}$ The com-

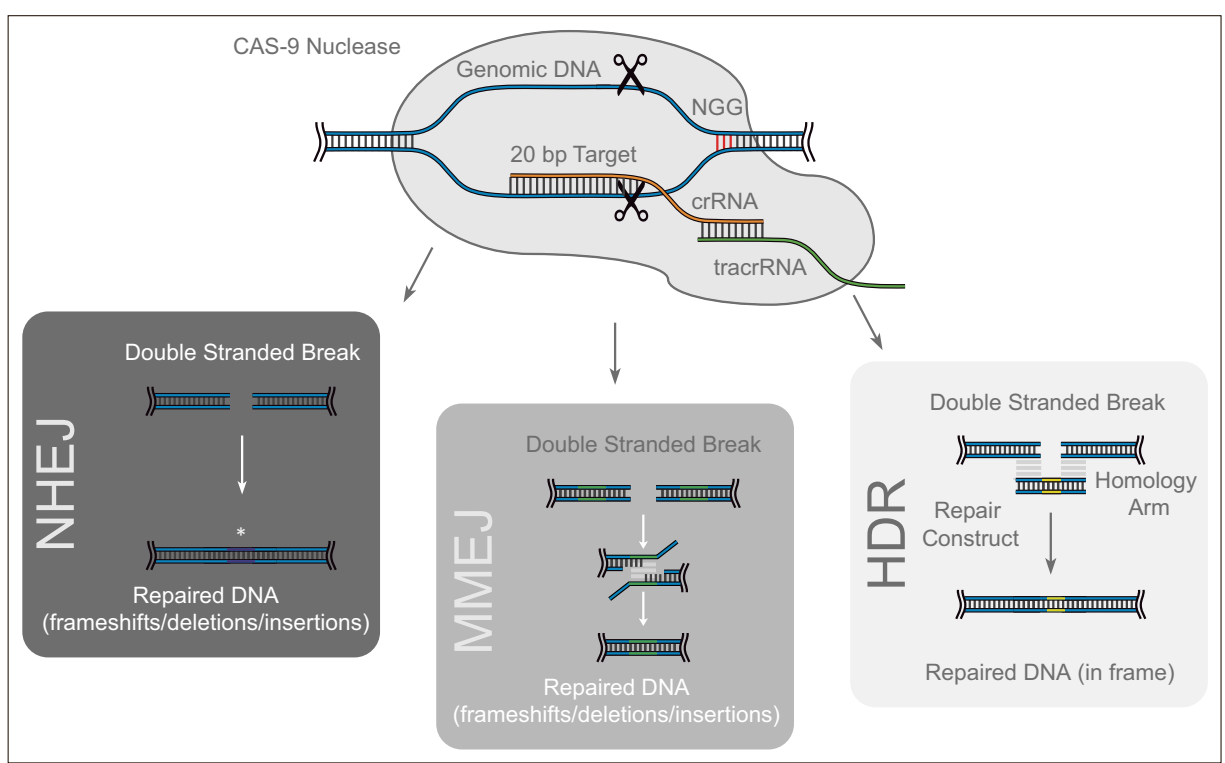

Fig. 1. An overview of CRISPR/Cas9 genome engineering via the exploitation of DNA repair mechanisms that follow the creation of double-stranded breaks. NHEJ, MMEJ, and HDR pathways can generate Indels for gene knockouts or the insertion of exogenous DNA sequences in the presence of a repair construct. 
bination of CRISPR/Cas9 technology with these mechanisms of DNA repair has enormous potential for biotechnological and therapeutic purposes, for example in the correction of disease-causing mutations. Yet despite application in a broad range of cell lines, primary cells, and even in vivo, the high efficiency delivery of CRISPR/ Cas9 machinery to cells ex vivo remains a key limitation. In this review, we compare different formats of both Cas9 and gRNA and discuss several state-of-the-art delivery methods while highlighting the respective advantages and challenges for ex vivo genome editing of mammalian cells.

\section{Formatting Cas9 for Delivery}

\subsection{Delivery of DNA Constructs}

The relatively large size of $S$. Pyogenes Cas9 (>1300 amino acids) creates unique challenges for intracellular delivery. Of primary concern when editing mammalian cells is the formatting of Cas9 in a manner that minimizes size for more efficient delivery yet preserves Cas 9 activity, restricts immune activation, and limits off target cleavage. Pioneering efforts in mammalian cells focused on encoding Cas9 in the form of DNA plasmids. ${ }^{[11,12]}$ This approach is attractive due to its simplicity; a single plasmid can be used to encode both the chimeric gRNA and the Cas9 under separate promoters. Another key advantage over other nuclease systems is the possibility to massively multiplex gene editing by the inclusion of multiple gRNAs expressed from the same plasmid.[13] Typically, the Cas9 is encoded as a fusion to a nuclear localization signal that mediates intranuclear transduction upon expression, thus allowing access to the genetic material of the cell. The delivery of plasmid DNA to cells either via standard chemical transfection or electroporation methods is very well established. In particular, the development of specialized electroporation systems, such as the Nucleofector $4 \mathrm{D}^{\mathrm{TM}}$ (Lonza) or the Neon ${ }^{\circledR}$ Transfection System (Thermofisher Scientific), have now made the transfection of even the most recalcitrant cell types, such as stem cells, a routine technique. However, for therapeutic engineering of mammalian cells with CRISPR/Cas9, plasmid DNA may not be the most suitable option as NHEJ mechanisms could potentially lead to plasmid integration in off-target locations or the activation of innate immune responses against double-stranded DNA. ${ }^{[14,15]}$ This could result in constitutive expression of the Cas9 from the genome and cause a higher frequency of unwanted off-target lesions.

A more complex alternative is to package the Cas9 DNA in single-stranded form within a non-integrating virus such as ad- eno-associated virus (AAV). ${ }^{[16]}$ The maximum packaging capacity for AAV is $\sim 4.5$ $\mathrm{kb}$, which makes combination of both the Cas9 and cognate gRNA into a single capsid challenging. Smaller Cas9 variants have been developed by truncation of the REC2 domain but suffer from a reduced efficacy by approximately $50 \% .^{[5]}$ Several systems have been developed to create split Cas9 enzymes, consequently allowing division between two separate AAV vectors. ${ }^{[17-19]}$ Again however, these systems often suffer from reduced activity as measured by the formation of Indels following the generation of DSBs. Possibly, the most promising avenue for AAV-based delivery lies in the use of significantly smaller Cas9 orthologs from other species. Ran et al. recently reported Cas9 isolated from $S$. aureus can edit mammalian genomes with similar efficiencies to S. Pyogenes Cas9 while being more than $1 \mathrm{~kb}$ shorter when encoded in DNA form. ${ }^{[20]}$ This advance allows Cas9 protein to easily be packaged within a single AAV capsid and has shown considerable promise in preclinical trials, particularly in the correction of Duchenne muscular dystrophy via the deletion of an aberrant exon. ${ }^{[21]}$

\subsection{Delivery of mRNA Constructs}

Another approach to reducing the size of Cas9 delivered to cells is to generate mRNA transcripts of the gene. Cas9 mRNA delivery has been widely adopted for the ex vivo modification of mammalian embryos by microinjection, ${ }^{[22-24]}$ and has more recently been applied to human somatic cell lines, ${ }^{[25]}$ or primary cells ${ }^{[26]}$ via electroporation. For the modification of embryos, impressive efficacies have been measured with Wang et al. reporting biallelic germline editing rates of up to $95 \%$ when a single gRNA was injected. Equally impressive was the reported knockout of endogenous CD3 in primary human T cells where after editing and fluorescence activated cell sorting (FACS), up to $85 \%$ of cells were negative for the antigen. ${ }^{[26]} \mathrm{A}$ primary advantage of using mRNA is the lack of integration of the Cas9 into the genome, although both mRNA stability and immunogenicity issues need to be addressed. The simplest approach to generate mRNA is by in vitro transcription from linearized DNA containing a T7 promoter. In order to improve stability, the Cas9 mRNA can be capped at the 5' end and a poly-A tail added to the $3^{\prime}$ end of the transcript. ${ }^{[27]}$ To prevent engagement of the immunoactivatory Toll Like Receptors 3, 7, and 8, mRNA may be chemically modified to create naturally occurring nucleoside derivatives pseudouridine and 5-methylcytidine. ${ }^{[28]}$

As with DNA, RNA constructs can also be delivered in viral form, most commonly in the form of integrase defective lentiviral particles. The lentivirus capsid has a substantially larger capacity for nucleic acid $(\sim 8 \mathrm{~kb})$ compared to AAV, and so it can be engineered to express both the Cas9 nuclease and up to four gRNAs simultaneously.[29,30] Thus far lentiviral systems have been predominantly applied for the creation of large genome wide loss-of-function libraries, which can be screened for gene function, for instance in cancer and cell ontogeny studies. ${ }^{[31]}$ For other applications, the combination of all components into a single system ensures more consistent levels of Cas9 expression, and is limited only by the range of cell types that can be successfully infected by lentivirus.

\subsection{Delivery of Cas9 \\ Ribonucleoproteins}

A more recent line of investigation has been to deliver ribonucleoproteins (RNPs), which are comprised of Cas9 protein precomplexed with gRNA. ${ }^{[25,32,33]}$ These studies suggest that delivering Cas9 in protein form leads to fewer observed off-target mutations than delivery via plasmid DNA. The reasons behind this observation are likely related to the kinetics of Cas9 availability, especially since the final levels of Indels detected after 72 hours are close to equal for plasmid and RNP delivery. Whereas protein Cas 9 begins to degrade immediately after delivery, Cas9 expressed from plasmids may not reach maximal levels for up to 24 hours, allowing more time for off-target lesions to develop. ${ }^{[32]}$ Schumann et al. used Cas9 RNPs to demonstrate high efficiency ex vivo editing in primary human $\mathrm{T}$ cells for knockout of the HIV co-receptor CXCR4. ${ }^{[34]}$ Up to $40 \%$ of targeted cells lost CXCR4 expression due to knockout, and when a repair template was supplied, approximately $20 \%$ of the population showed nucleotide correction in the CXCR4 gene, which was verified by deep sequencing of the locus.

Non-standard techniques for the delivery of Cas9 RNPs are an exciting new area of investigation. Lipid-based transfection reagents, which are most often used for generating complexes with DNA for transfection have now been explored for Cas9 RNP delivery. ${ }^{[35]}$ In this case, up to $80 \%$ of human U2OS EGFP reporter cells were negative for EGFP after delivery of Cas9 RNA combined with Lipofectamine 2000. Lipid technology can also be used for the generation of nanoparticle carriers of Cas9. By blending bioreducible lipid nanoparticles with negatively charged Cas9 gRNA, knockout of GFP in modified HEK cell lines could be observed at frequencies of $70 \% .^{[36]}$ Non-lipid carriers have also been applied to mimic vesiclelike structures for RNP delivery. Sun et $a l$. have reported the formation of 'nano- 
clews'; self-assembling DNA structures that bind Cas9 and are coated in PEI for transfection. ${ }^{[37]}$ Using this unique system, an Indel frequency of $28 \%$ was detected in human-derived U2OS cells.

The range of delivery options for Cas9 RNPs is further expanded upon by modification of the Cas9 protein termini to allow for covalent functionalization. For example, the addition of a C-terminal cysteine residue allows attachment of maleimide functionalized cell penetrating peptides (CPPs) via reaction with the free thiol group. ${ }^{[38]}$ When tested in HEK293T cells, the arginine rich Cas9-CPP fusion led to the formation of Indel mutations at a frequency of $14 \%$. Unfortunately, the CPPs in this system remain in the cells after delivery but next-generation CPPs based on reducible polydisulfide backbones are currently under evaluation for RNP delivery. ${ }^{[39]}$ As the use of CRISPR/Cas9 as a routine genome editing technique expands further, we expect even more novel RNP delivery avenues to be explored.

\section{Formatting the gRNA for Delivery}

For high efficiency genome editing using CRISPR/Cas9, not only is the successful delivery of Cas9 important, but also the associated gRNA. While both crRNA and tracrRNA can be delivered separately, the combination into a single chimeric gRNA considerably simplifies both design and delivery. This gRNA can either be expressed from a plasmid inside the cell or generated via in vitro transcription for loading into Cas9. Since unwanted Indel mutations can occur at off-target sites differing in up to five nucleotides, truncated gRNAs have been explored for higher fidelity. ${ }^{[40]}$ It was observed that shortening the complementary region of the gRNA by two or three nucleotides resulted in up to a 10,000-fold reduction in observed offtarget cleavage with minimal loss in Cas9 activity. Another concern is the inherent instability of RNA as compared to DNA. This can be somewhat mitigated through chemical modifications that extend the intracellular half-life by greatly reducing the rate of metabolic degradation. Hendle et $a l$. synthesized three different chemically modified sgRNAs by incorporating chemically altered nucleotides at the $5^{\prime}$ and $3^{\prime}$ termini. ${ }^{[41]}$ In particular, the 2'-O-methyl,3' phosphorothioate (MSP) modified single gRNA enhanced low efficiency genome editing rates in primary $\mathrm{T}$ cells and $\mathrm{CD} 34^{+}$ hematopoietic stem cells when co-delivered as a RNP or as pure RNA. Half-life was significantly increased ( $\geq 24 \mathrm{~h}$ ) for the chemically modified single gRNAs when compared to synthetic unmodified single gRNAs ( $\leq 4 \mathrm{~h})$. Unfortunately, the stepwise synthesis method used for production of these guides is not readily scalable and obtaining high purity modified guides ( 100 bases) remains a challenge. In order to address some of these limitations, Rahdar et al. described chemical synthesis of the crRNA alone ( 29 bases) containing a combination of phosphorothioate backbone modifications and the introduction of 2'-fluoro, 2'-O-methyl and S-constrained ethyl substitutions.[42] These synthetic guides can be combined with unmodified tracrRNA to reconstitute the active Cas9 complex.

\section{Combinatorial Delivery of Cas9 with Repair/Donor Constructs}

Improvements in the efficiency of CRISPR/Cas9 targeting in mammalian cells is making advanced genome-editing techniques readily accessible. In particu- lar, the supply of exogenously generated donor or repair templates allows for the insertion or correction of genes that may be many kilobases in size. Table 1 provides a representative summary of the progress made using CRISPR/Cas9 for the manipulation of human cells. The combination of the CRISPR/Cas9 components with DNAbased repair templates adds an additional degree of complexity in that the maximum availability of the Cas 9 nuclease versus the repair template should be considered. As the introduced donor/repair templates are typically non-replicative in mammalian cells, degradation begins immediately after delivery. Methods have been investigated to limit the rate at which degradation occurs by introducing multiple unnatural phospothiorate linkages at the 5 ' ends of both strands in dsDNA. ${ }^{[43]}$ Another possibility is to decouple the delivery of the repair template from that of the nuclease thus maximizing the quantities of each component. Such an approach has been used with meganucleases to enable extremely high efficiency editing in primary T cells ( $\sim 80 \%$ cassette insertion) by electroporation of the nuclease in mRNA form prior to donor template transduction using AAV. ${ }^{[15]}$ There is huge medical potential for CRISPR/Cas9 modification of mammalian cells, especially in the area of immunotherapy as evidenced by the CAR T cell revolution.

\section{Conclusions}

The rapid rise of CRISPR/Cas9 as a method for genome editing in mammalian cells has created a vast array of literature with numerous options for both format and delivery of the nuclease. Early studies used plasmid DNA as a simple and rapid method to deliver Cas9 for expression to the

Table 1. A survey of the approaches taken for the integration of increasingly large DNA constructs in human cells using CRISPR/Cas9.

\begin{tabular}{|c|c|c|c|c|c|c|}
\hline Reference & Cell Type & Cas9 Form & $\begin{array}{c}\text { Repair } \\
\text { Template Form }\end{array}$ & Insert Size (bp) & Mechanism & $\begin{array}{c}\text { Reported } \\
\text { Efficiency [\%] }\end{array}$ \\
\hline$[44]$ & HEK293 & RNP & ssODN & 3 & HDR & 60 \\
\hline$[43]$ & HEK293 & Plasmid & dsODN & 34 & NHEJ & $10-20$ \\
\hline$[45]$ & HEK 293 & Plasmid & Plasmid & $\sim 1350$ & MMEJ & $80^{\mathrm{a}}$ \\
\hline$[10]$ & HEK293 & Plasmid & PCR Prod & $\sim 1650$ & HDR & $50-66$ \\
\hline$[46]$ & RPE-1 & Plasmid & AAV & 2066 & HDR & $85^{\text {a }}$ \\
\hline$[7]$ & HEK293 & Plasmid & Plasmid & 4300 & NHEJ & 0.17 \\
\hline$[47]$ & LO2 & Plasmid & Plasmid & 4600 & HDR & 20 \\
\hline$[34]$ & LO2 & & & 34000 & NHEJ & 1.18 \\
\hline$[48]$ & T cells & RNP & ssODN & 12 & HDR & 20 \\
\hline$[47]$ & hPSC & Plasmid & Plasmid & 3000 & HDR & 0.001 \\
\hline
\end{tabular}

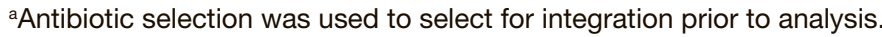


cell by standard electroporation or chemical transfection techniques. More recently, the field has moved to explore alternative formats as evidence has emerged that plasmid DNA can potentially be integrated via NHEJ mechanisms into the cells being engineered. While some systems, such as $\mathrm{AAV}$, hold promise due to high infection efficiency and clinical trial data that support a favorable safety profile, delivery of Cas9 as an RNP is attractive for ex vivo cellular engineering. Several studies have now reported a reduced frequency of observed off-target effects when compared to delivery of Cas9 by plasmid DNA. As the applications for CRISPR/Cas9 editing mature and head towards in vivo studies in humans, reducing off-target effects will be critical in ensuring the safety of next-generation gene therapies. As a complement to Cas9 delivery, consideration must be given to the format of the gRNA being delivered. Protecting the gRNA from metabolic degradation pathways can be achieved through chemically synthesized backbone and base modifications. Scaling high purity production of these guides remains challenging but will undoubtedly improve over time. In future, we expect that further investigations into optimal delivery strategies and combinations of CRISPR/Cas9 component formats will allow high efficient editing to be tailored to any cell type.

\section{Acknowledgment}

We thank the National Centre of Competence in Research (NCCR) Molecular Systems Engineering, administered by the Swiss NSF, for financial support. Gratefully acknowledged is additional financial support from the ETHZ, the University of Geneva, and the Swiss NSF.

\section{Received: March 21, 2016}

[1] M. Jinek, K. Chylinski, I. Fonfara, M. Hauer, J. A. Doudna, E. Charpentier, Science 2012, 337, 816.

[2] R. Barrangou, C. Fremaux, H. Deveau, M. Richards, P. Boyaval, S. Moineau, D. A. Romero, P. Horvath, Science 2007, 315, 1709.

[3] G. Gasiunas, R. Barrangou, P. Horvath, V. Siksnys, Proc. Natl. Acad. Sci. USA 2012, 109, E2579.

[4] P. Mali, L. Yang, K. M. Esvelt, J. Aach, M. Güell, J. E. DiCarlo, J. E. Norville, G. M. Church, Science 2013, 339, 823.

[5] H. Nishimasu, F. A. Ran, P. D. Hsu, S. Konermann, S. I. Shehata, N. Dohmae, R. Ishitani, F. Zhang, O. Nureki, Cell 2014, 156, 935.

[6] M. Bibikova, M. Golic, K. G. Golic, D. Carroll, Genetics 2002, 161, 1169.
[7] R. Bachu, I. Bergareche, L. A. Chasin, Biotechnol. Bioengin. 2015, 112, 2154.

[8] T. Kent, G. Chandramouly, S. M. McDevitt, A. Y. Ozdemir, R. T. Pomerantz, Nature Struct. Mol. Biol. 2015, 22, 230.

[9] M. Bibikova, D. Carroll, D. J. Segal, J. K. Trautman, J. Smith, Y. G. Kim, S. Chandrasegaran, Mol. Cell. Biol. 2001, 21, 289.

[10] Van Trung Chu, T. Weber, B. Wefers, W. Wurst, S. Sander, K. Rajewsky, R. Kühn, Nat. Biotechnol. 2015, 33, 543.

[11] L. Cong, F. A. Ran, D. Cox, S. Lin, R. Barretto, N. Habib, P. D. Hsu, X. Wu, W. Jiang, L. A. Marraffini, F. Zhang, Science 2013, 339, 819.

[12] M. Jinek, A. East, A. Cheng, S. Lin, E. Ma, J. Doudna, D. Weigel, eLife Sciences 2013, 2, e00471.

[13] T. Sakuma, A. Nishikawa, S. Kume, K. Chayama, T. Yamamoto, Sci. Rep. 2014, 4, 5400.

[14] P. K. Mandal, L. M. R. Ferreira, R. Collins, T. B. Meissner, C. L. Boutwell, M. Friesen, V. Vrbanac, B. S. Garrison, A. Stortchevoi, D. Bryder, K. Musunuru, H. Brand, A. M. Tager, T. M. Allen, M. E. Talkowski, D. J. Rossi, C. A. Cowan, Cell Stem Cell 2014, 15, 643.

[15] B. D. Sather, G. S. Romano Ibarra, K. Sommer, G. Curinga, M. Hale, I. F. Khan, S. Singh, Y. Song, K. Gwiazda, J. Sahni, J. Jarjour, A. Astrakhan, T. A. Wagner, A. M. Scharenberg, D. J. Rawlings, Sci. Transl. Med. 2015, 7, 307ra156.

[16] E. Senís, C. Fatouros, S. Große, E. Wiedtke, D. Niopek, A. K. Mueller, K. Börner, D. Grimm, Biotechnol. J. 2014, 9, 1402.

[17] E. J. Fine, C. M. Appleton, D. E. White, M. T. Brown, H. Deshmukh, M. L. Kemp, G. Bao, Sci. Rep. 2015, 5, 10777.

[18] A. V. Wright, S. H. Sternberg, D. W. Taylor, B T. Staahl, J. A. Bardales, J. E. Kornfeld, J. A. Doudna, Proc. Natl. Acad. Sci. USA 2015, 112, 2984.

[19] B. Zetsche, S. E. Volz, F. Zhang, Nat. Biotechnol. 2015, 33, 139.

[20] F. A. Ran, L. Cong, W. X. Yan, D. A. Scott, J. S Gootenberg, A. J. Kriz, B. Zetsche, O. Shalem, X. Wu, K. S. Makarova, E. V. Koonin, P. A. Sharp, F. Zhang, Nature 2015, 520, 186.

[21] C. E. Nelson, C. H. Hakim, D. G. Ousterout, P. I. Thakore, E. A. Moreb, R. M. C. Rivera, S. Madhavan, X. Pan, F. A. Ran, W. X. Yan, A. Asokan, F. Zhang, D. Duan, C. A. Gersbach, Science 2016, 351, 403.

[22] T. Hai, F. Teng, R. Guo, W. Li, Q. Zhou, Cell Res. 2014, 24, 372.

[23] Y. Niu, B. Shen, Y. Cui, Y. Chen, J. Wang, L. Wang, Y. Kang, X. Zhao, W. Si, W. Li, A. P. Xiang, J. Zhou, X. Guo, Y. Bi, C. Si, B. Hu, G. Dong, H. Wang, Z. Zhou, T. Li, T. Tan, X. Pu, F. Wang, S. Ji, Q. Zhou, X. Huang, W. Ji, J. Sha, Cell 2014, 156, 836.

[24] H. Wang, H. Yang, C. S. Shivalila, M. M. Dawlaty, A. W. Cheng, F. Zhang, R. Jaenisch Cell 2013, 153, 910.

[25] X. Liang, J. Potter, S. Kumar, Y. Zou, R Quintanilla, M. Sridharan, J. Carte, W. Chen, N. Roark, S. Ranganathan, N. Ravinder, J. D. Chesnut, J. Biotechnol. 2015, 208, 44.

[26] M. J. Osborn, B. R. Webber, F. Knipping, C. L. Lonetree, N. Tennis, A. P. DeFeo, A. N. McElroy, C. G. Starker, C. Lee, S. Merkel, T. C. Lund, K. S. Kelly-Spratt, M. C. Jensen, D. F. Voytas, C. Von Kalle, M. Schmidt, R. Gabriel,
K. L. Hippen, J. S. Miller, A. M. Scharenberg, J. Tolar, B. R. Blazar, Mol. Therapy 2015, 24, 570.

[27] B. Shen, J. Zhang, H. Wu, J. Wang, K. Ma, Z. Li, X. Zhang, P. Zhang, X. Huang, Cell Res. 2013, 23, 720 .

[28] K. Karikó, H. Muramatsu, F. A. Welsh, J. Ludwig, H. Kato, S. Akira, D. Weissman, Mol. Therapy 2008, 16, 1833.

[29] N. E. Sanjana, O. Shalem, F. Zhang, Nat. Meth. 2014, 11, 783.

[30] A. M. Kabadi, D. G. Ousterout, I. B. Hilton, C. A. Gersbach, Nucleic Acids Res. 2014, 42, e147.

[31] O. Shalem, N. E. Sanjana, E. Hartenian, X. Shi, D. A. Scott, T. S. Mikkelsen, D. Heckl, B. L. Ebert, D. E. Root, J. G. Doench, F. Zhang, Science 2014, 343, 84.

[32] S. Kim, D. Kim, S. W. Cho, J. Kim, J.-S. Kim, Genome Res. 2014, 24, 1012.

[33] S. Lin, B. T. Staahl, R. K. Alla, J. A. Doudna, D. Weigel, eLife Sciences 2014, 3, e04766.

[34] K. Schumann, S. Lin, E. Boyer, D. R. Simeonov, M. Subramaniam, R. E. Gate, G. E. Haliburton, C. J. Ye, J. A. Bluestone, J. A. Doudna, A. Marson, Proc. Natl. Acad. Sci. USA 2015, 112, 10437.

[35] J. A. Zuris, D. B. Thompson, Y. Shu, J. P. Guilinger, J. L. Bessen, J. H. Hu, M. L. Maeder, J. K. Joung, Z.-Y. Chen, D. R. Liu, Nat. Biotechnol. 2015, 33, 73.

[36] M. Wang, J. A. Zuris, F. Meng, H. Rees, S. Sun, P. Deng, Y. Han, X. Gao, D. Pouli, Q. Wu, I. Georgakoudi, D. R. Liu, Q. Xu, Proc. Natl. Acad. Sci. USA 2016, 201520244, DOI: 10.1073/pnas.1520244113.

[37] W. Sun, W. Ji, J. M. Hall, Q. Hu, C. Wang, C. L. Beisel, Z. Gu, Angew. Chem. Int. Ed. 2015, 54, 12029.

[38] S. Ramakrishna, A.-B. Kwaku Dad, J. Beloor, R. Gopalappa, S.-K. Lee, H. Kim, Genome Res. 2014, 24, 1020 .

[39] G. Gasparini, S. Matile, Chem. Commun. 2015, $51,17160$.

[40] Y. Fu, J. D. Sander, D. Reyon, V. M. Cascio, J. K. Joung, Nat. Biotechnol. 2014, 32, 279.

[41] A. Hendel, R. O. Bak, J. T. Clark, A. B. Kennedy, D. E. Ryan, S. Roy, I. Steinfeld, B. D. Lunstad, R. J. Kaiser, A. B. Wilkens, R. Bacchetta, A. Tsalenko, D. Dellinger, L. Bruhn, M. H. Porteus, Nat. Biotechnol. 2015, 33, 985.

[42] M. Rahdar, M. A. McMahon, T. P. Prakash, E. E. Swayze, C. F. Bennett, D. W. Cleveland, Proc. Natl. Acad. Sci. USA 2015, 112, E7110.

[43] S. Q. Tsai, Z. Zheng, N. T. Nguyen, M. Liebers, V. V. Topkar, V. Thapar, N. Wyvekens, C. Khayter, A. J. Iafrate, L. P. Le, M. J. Aryee, J. K. Joung, Nat. Biotechnol. 2015, 33, 187.

[44] C. D. Richardson, G. J. Ray, M. A. DeWitt, G. L. Curie, J. E. Corn, Nat. Biotechnol. 2016, 34, 339.

[45] S. Nakade, T. Tsubota, Y. Sakane, S. Kume, N. Sakamoto, M. Obara, T. Daimon, H. Sezutsu, T. Yamamoto, T. Sakuma, K.-I. T. Suzuki, Nat. Commun. 2014, 5, 5560.

[46] M. Kaulich, Y. J. Lee, P. Lönn, A. D. Springer, B. R. Meade, S. F. Dowdy, Nucleic Acids Res. 2015, 43, e 45 .

[47] X. He, C. Tan, F. Wang, Y. Wang, R. Zhou, D. Cui, W. You, H. Zhao, J. Ren, B. Feng, Nucleic Acids Res. 2016, DOI: 10.1093/nar/gkw064.

[48] F. T. Merkle, W. M. Neuhausser, D. Santos, E. Valen, J. A. Gagnon, K. Maas, J. Sandoe, A. F. Schier, K. Eggan, Cell Rep. 2015, 11, 875. 